\title{
Introduction: E Pluribus Unum
}

The 12 papers in this special issue of Journal of Biosciences are based on talks given at a discussion meeting on the theme 'Individuals and Groups' that was held in Almora from 22 to 31 May 2012. About 50 people took part, all of them as discussants and approximately half also as speakers. Not everyone who spoke contributed an article; the shortfall was made up partially by some who were unable to attend but agreed to write. These papers are a fair representation of the range of viewpoints, though not systems, covered at Almora. The aim of the meeting was to explore the evolutionary basis of group behaviour in biology and discuss commonalities and differences between diverse viewpoints. A question that might provide a common theme had been suggested to the speakers: To what extent can one account for group behaviour in terms of the properties of the constituent units as exhibited when they are isolated, and to what extent does one need to invoke group-level, 'emergent' traits? As will be seen, the question is addressed in different ways, some overlapping and others in apparent conflict, by the authors of this special issue. Even though the responses concern group behaviour in biological systems, many contain indications of how the individual versus group issue is confronted in physics and chemistry. Within biology, groups at different levels of organization - genes, proteins, metabolic pathways, cells, organisms and species - are considered. Before introducing the papers in this issue, we offer our own perspective on individuals and groups.

Until the influential intervention of Darwin and Wallace, biologists tended to adopt either a functionalist approach to the explanation of living systems, typically one (as with Cuvier) in which the purposes the functional units served were stipulated by a Creator, or alternatively, a structuralist approach (as with Geoffroy Saint-Hilaire), somewhat akin to physics and chemistry, in which organizational properties were assumed to be intrinsic to the constituent materials, and function was relatively incidental. The Darwinian revolution advanced the functionalist tradition but shifted the focus on why the traits of living organisms were what they are away from the creationist mode. It put forward an account that involved extrinsic factors - the demands of the environment and natural selection, following an internal but undirected event, random variation (later tied to genetic change). Current thinking has led to a resurgence of interest in structuralist approaches, but has attempted to incorporate the major insights of a century of Darwinian functionalism.

Functional (or 'proximate' or 'how') explanations in biology lean on immediately preceding events and are typically based on phenomenological physics and chemistry regarding mechanism, if not organization. Biology stands out from the other natural sciences in that it also demands a qualitatively different sort of explanation, one that is called historical or 'ultimate'. Ultimate explanations derive from the historical fact of organic evolution. Cosmology and the evolution of the chemical elements also involve history, but one that is many levels removed from the perceptible physical properties of present-day forms. It would seem odd if someone were to say 'Nothing in physics makes sense except in the light of evolution', whereas the corresponding statement was proposed by Dobzhansky as a tenet of biology. The difference is that the properties of organisms tend to be rationalized by their histories, while physical properties and laws tend to be treated as givens.

In the Darwinian framework, ultimate explanations are based on natural selection, which endows them with a flavour that is unique to biology. In turn, natural selection is based on variations in organismal traits that derive from the working of genes within living matter; it acts on organisms and claims to diversify lineages and modify species. A broad consensus developed around the 1950s regarding how evolution 
worked. Known as the neo-Darwinian or Modern Synthesis, it depended largely though not exclusively on natural selection. We are still far from understanding how the mutual interactions of a multitude of genes with the microenvironments assembled by gene products, and with the broader external environment, give rise to an individual organism, or even a single cell; in short, how genotypes are related to phenotypes.

The problem becomes more acute when it comes to groups of organisms, because here even the single organism-centred view may be questionable, all the more so when group members interact strongly and cooperate with one another. Recognition of this fact has fostered a whole array of Modern Synthesisconnected proposals that seek to bridge the gap between gene-centred and organism-centred views. Their essence goes back to Darwin himself, who typically did not confine himself to a single explanation for the evolution of group behaviour. The proposals were fleshed out later by Haldane, Fisher and Wright, and more recently by Hamilton, Trivers and DS Wilson. By an insightful arrangement of the factors on which trait variation might depend, Price formulated what is in principle an all-encompassing description of cooperation. The description is in terms of a scheme that incorporates the overall change in fitness resulting from both within-group and between-group effects. His scheme explicitly accounts for within-group effects (that disfavour cooperation between individuals) and between-group effects (that favour cooperation), and shows when the latter can override the former. Modifications can incorporate the possible role of kinship or genetic similarity in social behaviour.

These strategies are powerful, but they also have inherent limitations. An evolutionary explanation has to account for the two characteristic features of living matter. The first is adaptation: '[Living creatures] have morphologies, physiologies and behaviours that appear to have been carefully and artfully designed to enable each organism to appropriate the world around it for its own life' (Lewontin, 1978). The second is that as material entities, microbes, plants and animals seem to be complex, intricate and extremely improbable. It follows that there must be a means whereby standard physical and chemical processes can give rise to outcomes that are both highly unlikely and, over the long run, unpredictable. This conclusion is at odds with the simple-minded extrapolation, based on the success of Newtonian thinking, that natural processes must lead to causally transparent outcomes. When Darwin and Wallace put forward natural selection acting on individuals as an explanation of evolution, it appeared to many that at one stroke they had accounted for adaptation, diversity and complexity in the living world. Natural selection has provided the most widely accepted framework for evolutionary thinking ever since, but it has always had its critics, particularly with respect to the origins of novel forms.

Both Darwin and Wallace did acknowledge that processes other than natural selection could be lie behind evolutionary change and evolutionary stability, and since their time the way we look at evolution has been enriched by a better understanding of heredity and variation, limitations to the scope of natural selection, and recognition of the range of possibilities open to physical and chemical systems. The papers in this issue highlight different aspects of this improved understanding in the context of collective properties or group behaviours exhibited at different levels of organisation.

In trying to explain the behaviour of composite systems, a question that crops up is how far one can depend solely on the properties of the units that make up the system. At what stage does it become necessary to bring in system-level properties as descriptors? Physics has been the most successful of the natural sciences in grappling with such questions through a range of tools including statistical mechanics and many-body theory. While quantum mechanics provides their ultimate justification, in many physical situations (e.g. where the identity of particles plays no fundamental role), a non-quantum-mechanical description is adequate for all practical purposes.

On the whole, the standpoint of chemistry, especially when it is seen as the study of transformations, has been to focus on phenomena. Rules of convenience are utilized at various levels of description (e.g. the law of mass action, or the Michaelis-Menten equation for enzyme kinetics). This is all the more true of biology. Except where they concern behaviour at the molecular level, in which case there is a strong grounding in physics and chemistry, theories in biology are almost entirely phenomenological. Mendel's laws of genetics constitute the pre-eminent example.

Instances of more than one potential or actual unit of reproduction functioning as part of a larger whole (which is composed of similar or dissimilar units) are ubiquitous in biology. The unit can be one or another member of a loosely nested hierarchy: a gene or DNA sequence, a chromosome, an intracellular organelle, a cell, a tissue (organ) or an entire organism - a microbe, plant or animal. In many cases, the whole displays 
emergent properties, that is, modes of functioning that are not seen, or sometimes not possible, among its constituent units; the study of such properties in microbial populations and biofilms is a recent trend. One attempts to account for the property in question in terms of those that the units exhibit when examined separately, and when that does not work, to look for other explanations. The phenomenon may reflect a colligative property, namely, one that depends on number or size. Or, an individual may gain an advantage simply by being part of the crowd, so to speak. In the process, the group may develop a spatial structure that derives from nothing more than each member acting independently and attempting to do the same thing (e.g. staying as far from the periphery as possible), as Hamilton pointed out. When a 'colligative' explanation does not suffice, one looks for an explanation that involves, over and above the properties of the individual units, the interactions that occur between them. The restriction to proximate causes remains.

Coming to the papers themselves, we draw attention now to what we take to be the distinguishing messages, but readers are likely to draw additional lessons from these essays.

Cihan Saçlığlu, Önder Pekcan and Vidyanand Nanjundiah compare and contrast physical, chemical and biological systems. The theories of physics rest on laws, quasi-epigrammatical and largely mathematical assertions of enormous scope. At its most basic level, group behaviour involves the laws of symmetry dictated by the quantum mechanical properties of identical elementary particles. All the way from the microscopic to the macroscopic domain, its manifestations depend on the discreteness of Planck's constant. Phase transitions typify cooperative group behaviour. One of them, the sol-gel transition, occurs in chemical systems and living cells. In the physical and chemical systems that are usually studied, proximate causes suffice to account for cooperative behaviour in groups, whereas in living systems one must more often take recourse to historical contingencies and other distant causes. In a parallel fashion, the behaviour of organisms in groups is influenced by physics (e.g. size), chemistry (e.g. adhesion), biology (e.g. relatedness) and of course evolution.

Multicellular development is the supreme example of group behaviour; three papers highlight different implications that can be drawn from it.

Patrick Bateson nicely summarises the task that must be confronted: reductionist biology has succeeded in deepening our knowledge of how to take things apart; now one must begin to think of how to bring the parts together. It is important to study developmental change - epigenetic processes - if we wish to understand what makes a group of cells behave as one unit and, by extension, what makes a group of animals behave as one unit. In the case of a multicellular organism, genetic identity between cells assures shared genetic interests; in other cases, the advantage of size may be enough to favour cooperation out of self-interest. But there may be group-level adaptations that are not based on either factor. In particular, if individual behaviour is not a Mendelian trait but depends on interactions between gene products, cooperation between individuals may not hinge on the familiar cost-benefit-relatedness inequality. Also, groups made up of genetically unrelated components, such as lichens, can display 'parent-offspring' correlations in which genetic variation in one component influences the reproductive success of the other.

Scott Gilbert takes off from the numerous recorded instances of obligatory symbiotic associations that are present in metazoan bodies and have a decisive influence on the phenotype of the host (e.g. on the immune system). He makes the radical suggestion that we should begin to look at organisms not merely as groups of genetically identical cells but as groups of multicellular groups of cells ('holobiont', for the integrated organism consisting of host cells and persistent symbionts). The conventional picture of development gets a twist here: the course of development from fertilization onwards is underpinned by reciprocal interactions between the heterogeneous groups. Reminiscent of what is seen in lichens, genetically variant symbionts can be associated with heritable variations in host fitness. Once again, by implication the associations are proof that cooperative group life need not depend on shared genes. Gilbert's view of group behaviour as exhibited by holobionts has intriguing parallels to group behaviour in asexual organisms that are considered to be members of the same species.

Marta Linde-Medina and Stuart Newman cite different examples of multicellular development to illustrate that evolution can (i) be slow and gradual (as natural selection via the accumulation of minor changes would demand), (ii) depend (to different extents in the history of multicellular forms) on interaction of the developing organism with the environment (unlike in conventional evolutionary theory), and (iii) lead to abrupt qualitative changes of form (something inconsistent with the predictions of natural selection) if feedbacks or other complex interactions are present. In the last case, cells which come pre-adapted with 
the requisite property (e.g. motility and adhesivity) could be triggered, in a collectivity, or in physically interacting collectivities, to form a novel multicellular structure by a small genetic or environmental change. Contrary to conventional selectionist thinking, micro- and macro-evolution may therefore be based on different principles according to these authors. Both Bateson and Linde-Medina \& Newman cite genetic assimilation as a possible process through which an evoked environmentally induced change remains heritable in the absence of the environmental stimulus.

The next two papers deal with multicellularity that comes about in an unusual manner: not because the clonal progeny of a zygote stay together, but because physically separated cells, which may or may not have a higher than average probability of sharing genes via recent common descent, come together.

Dominika Włoch-Salamon draws attention to the fact that there can be situations in which single gene effects and shared genes may underlie cooperative traits. She discusses the explicit example of genetic variation in a single cell influencing a trait that impinges on social behaviour, interestingly, in brewer's yeast - an organism that was long thought of (wrongly, as she points out) as constitutively unicellular. The traits in question are the ability to flocculate, invertase production, the release of a toxin by a cell that is itself resistant to the toxin and, finally, the seemingly maladaptive phenomenon of 'programmed' cell death (apoptosis). A case can be made for each of these to be disfavoured at the individual level and favoured in groups. But the associated costs and benefits are not always obvious. Also, the important question of whether the group can be subverted by an individual that benefits from belonging to a group without incurring the cost of membership (e.g. by producing flocculin) remains open. Finally, Włoch-Salamon considers group-level traits that are present in yeast and other microorganisms including Escherichia coli that have recently been discovered to have a social life. Are these traits better understood as examples of convergent evolution, i.e. as adaptations that have evolved independently in different groups under comparable environmental pressures (or opportunities), or as generic, 'automatic' consequences of the pre-existing properties of single cells?

Silvia De Monte and Paul Rainey continue with the theme of facultative sociality. Their focus is on the origin of group behaviour, which they attribute plausibly to the formation of ephemeral groups by cells that are also capable of living freely (for an example, see photographs on the Cover). Participation in a group is viewed as a transitory and non-reproductive phase in the life cycle of a cell. How do the standard requirements for evolution by natural selection (the existence of entities that display heritable variation in traits that are correlated with lifetime reproductive fitness) fare under these circumstances? If groups do not reproduce as groups, and the composition of a group in one generation is only weakly linked (via heredity) with the composition of a group in the next, can group-level traits contribute to fitness at all (and so conceivably evolve by natural selection)? The first question is answered by saying that strict parent-offspring relationships are not necessary; it is sufficient that some individuals in a group in one generation are ancestors to some individuals in a group in the following generation. The second question is answered in the affirmative with the explicit example of a model of motile cells that vary in their ability to form groups by adhering to one another.

The following two papers explore the requirements for coordinated behaviour among and within cells.

Bahram Houchmandzadeh emphasizes the importance of looking closely at the consequences of a null evolutionary hypothesis before assuming that selection must be at work. He demonstrates that randomly occurring birth and death processes, which by their very nature change the population size by one unit, are sufficient to account for many group-level phenomena. They include spatial clustering and the distribution of species abundances in the MacArthur-Wilson island model of ecology. Surprisingly, an analogous neutral model also suffices to show that, under specified conditions, it is more likely for a trait that is individually disadvantageous but socially advantageous to spread through a group, than the other way round.

Göker Arpağ and Ayșe Erzan examine regulatory gene and protein interactions from a physicalist viewpoint. They show that analogously to the preferred phase (gas, liquid or solid) of a physical system, what looks like an evolved adaptation in a system of biological molecules may simply be statistically the most probable configuration of a system that arises from 'self-organization'. Networks of interactions involving regulatory RNA molecules supposedly exist to ensure that the right genes are expressed at the right time and in the right place. But a network of this sort may fall in place automatically if a certain set of RNA molecules which may have been there for completely different reasons happen to coexist in a cell at the same time. Their simultaneous existence, together with the combinatorial possibilities opened up by 
binding, leads to outcomes that, in hindsight, appear to be specific adaptations 'for the purpose' of gene regulation but are in fact due to pre-adaptations. Thus, Arpağ and Erzan too emphasize that one must first test the appropriate null hypothesis before invoking natural selection.

Carlos Sonnenschein, Ana Soto, Annapoorni Rangarajan and Prakash Kulkarni debate how to view carcinogenesis, conventionally thought of as an example of narrow group interest (that of the cancer cells) overriding the broader good (that of the organism). By adopting dramatically different viewpoints, the questions they pose encapsulate almost all the other debates that dominated the Almora meeting. The very first echoes a common theme in sociological analyses of pathological behaviour: should one pinpoint the origin of cancer to an aberrant single cell, or should the society of cells be held responsible? If the former, which are more important, 'focal' genetic changes or 'systemic' changes in the interlocked networks of gene products? If the latter, can one draw a meaningful distinction between the relevant social group and its environment? The theoretical frameworks used to address these questions are the tissue organization field theory (CS and AS), the cancer stem cell hypothesis (AR), and the concept of intrinsically disordered proteins (PK). Their paper is explicit about the epistemological and ontological issues that lie behind scientific debates but are often ignored. How does one decide what should be the basic elements of a theory? When is it permissible to modify a theory to account for exceptions and when is it no longer tenable? On what basis does one say that two theories are different? May one merge theories, and if so, under what circumstances? With all their differences the authors give the impression of being in agreement on one point: carcinogenesis may not lend itself to a unitary explanation.

Ellen Clarke, Telmo Pievani and George Katsiaficas round off the special issue with three papers that deal largely with philosophy. Clarke teases out the multi-level hierarchies inherent in group phenomena. She points out the usefulness of viewing group behaviour as analogous to a phase transition. A phase transition in physics is underpinned by the transfer of causal agency from the subunit (individual) to the aggregate (group); the corresponding transfer in the evolution of group behaviour in biology is that of heritable variation between groups vis-à-vis the individuals within a group. This way of posing the issue makes it natural to use Price's formalism for the partitioning of variance. Clarke joins Włoch-Salamon and De Monte \& Rainey in alluding to stickiness as an example of an individual-level trait that lends itself to exploitation for a group-level adaptation. To begin with, the trait is a pre-adaptation. By virtue of its capacity to induce an 'alignment of fitness' among the constituent individuals, it is a prerequisite for the action of higher-level selection. By sharply scrutinizing the long-standing (and recently revived) debate on kin selection and group selection, and exposing the sterility of dichotomous framings, Pievani performs a major service. He convincingly argues that the exclusivity insisted on by adherents of one or the other hypothesis is chimerical. Nature is messy; explanations that combine what seem to be different approaches are not just pragmatic, they are inevitable. According to Pievani, it is often forgotten that Darwin advocated precisely such a plurality of views. Pievani and Clarke agree, moreover, that multi-level selection theory is the appropriate way to update the concept of natural selection.

Katsiaficas builds his discussion around the analysis of human cooperation carried out by the 14th century scholar Ibn Khaldun, who has claims to have initiated the scientific study of human society and, in his consideration of the emergence of humans from 'the world of monkeys' through a process in which 'species become more numerous', aspects of evolutionary theory. For him, group solidarity, exemplified most strongly by families, hinged on blood relationships. Nomadic existence fostered the tendencies advantageous to group life, whereas a sedentary urban existence was detrimental to it, a notion that is worth contrasting with ecological theories of social behaviour. Finally, as characterized by Katsiaficas, Ibn Khaldun's view of the emergence of human society being accompanied by 'human beings becoming who they in essence are', echoes the group-individual dialectic seen at all levels throughout this collection.

In a nutshell, the papers in this issue offer a range of explanations for the evolution of cooperative behaviour in biological groups - for how, as the title of this Introduction states, one emerges out of many. They include 'old fashioned' natural selection acting on individual members of the group; natural selection fostered by shared genes; multi-level selection; pre-adaptation and self-organization; and reciprocal dynamical interactions between individuals, groups and the environment. A group may consist of individuals between whom genes are rarely or never exchanged. The ontogeny of group behaviour is 
likely to offer strong clues regarding its underlying basis. Finally, it is important to guard against the temptation of single answers to evolutionary questions; the uniqueness of the group may be as salient as the uniqueness of the individual.

\section{Acknowledgements}

The efforts of many people made this Special Issue possible. The International Centre for Theoretical Sciences supported the discussion meeting without any of the tiresome bureaucratic demands that one usually takes for granted. The director and staff of the G B Pant Institute for Himalayan Environment and Development were our hosts at Almora. They located a suitable venue, arranged for people to be ferried across large distances and, with patience and good humour, made sure that the proceedings went smoothly from day to day. The Editor of the Journal of Biosciences agreed readily to publish the articles. To all of them, in particular Spenta Wadia, Lok Man Singh Palni, Ranbeer Rawal and Durgadas Kasbekar, and to those who have painstakingly critiqued the articles in this issue, we offer our heartfelt thanks. Krithi Nandimath is acknowledged for efficient and cheerful assistance before and during the meeting. Jai Benjamin and Sushila Rajagopal of Journal of Biosciences were as helpful and accommodating as one would wish the staff of a journal to be.

Vidyanand Nanjundiah ${ }^{l}$ and StUART A Newman ${ }^{2}$
${ }^{1}$ Centre for Human Genetics,
Electronic City (Phase I)
Bangalore 560100,
India
(Email, vidya@ces.iisc.ernet.in)
${ }^{2}$ New York Medical College Valhalla
10595 New York, USA
(Email, newman@nymc.edu)

